\title{
On the Question of Analyzing of the Iteratively Integrating Measurement Converter
}

\author{
Igor Sergeyev \\ Aviation Computer-Integrated Complexes Department \\ National Aviation University \\ Kyiv, Ukraine \\ sergeyevi@i.ua
}

\begin{abstract}
Generalized structural scheme of the measurement converter which uses iteratively integrating conversion method is described. Analysis of its conversion equation and errors for the case where the input variable $X=X(t)$ is not a constant, while the input values $Z 1, Z 2$ and output value $Y$ are constant, was produced. Basic expressions for the calculation are listed.
\end{abstract}

Keywords-generalized structural scheme; iteratively integrating conversion method; conversion equation; integrator; iterative additive correction of errors; dynamics of the iterative process, steady state

\section{INTRODUCTION}

As known, in the technological information systems related to the collection, processing and transmission of information measuring converters are essential. The metrological parameters of the entire measuring system are often largely determined by the metrological parameters of these measuring converters. The achievement of linearity of their functions transformation is very important for measuring converters application in such systems, and consequently for independence of static transformative characteristics from value of transformed quantity. Reduction of level of error measuring converters often plays a decisive role in solving the problem of creation of information systems. Among the many methods of increasing the accuracy of measuring converters structural methods are very important [1]. Structural methods of improving the accuracy include use of circuits in which it is possible to eliminate or reduce the influence of some of the errors most unstable blocks and elements for the total error of the device. A significant place among structural methods to increase the accuracy of measuring converters occupy iterative methods of error correction.

Combining methods of integrating conversion and additive iterative correction of errors has generated a method called by the author [2] - [4] iteratively integrating conversion method and devices using this method - iteratively integrating converters (IIC). As known, many of iteratively integrating converters possess high metrological parameters.

Analysis of the generalized structural scheme of iteratively integrating transducers for the case when the input values $X$, $Z 1, Z 2$ and output value $Y$ are constant values was made by the author in [4].

\section{PRoblem StATEMENT}

However, this case does not fully describe the operation of the converter.

It is of interest to consider the case where the input variable $X=X(t)$ is not a constant, while in the input values $Z 1, Z 2$ and output value $Y$ are constant.

\section{SOLUtion OF THE PROBlem}

Simplified generalized structural scheme of iteratively integrating converter shown in Fig. 1, which was offered by the author in [2]. Here in the simplest case $X$ is the input value; $Y$ is the output value; $\mathrm{OC}$ is output converter, $\mathrm{BC}$ is back converter; $\mathrm{K}_{\mathrm{BC}}$ is transfer coefficient of $\mathrm{BC}$; $\mathrm{SW} 1$ and SW2 are switches, $\mathrm{SH}$ is sample-and-hold device; $T 1$ is time interval of integration of the input variable $X ; T 2$ is time interval of integration of the value $\beta Y$. The values of $T 1$ and $T 2$, along with $X$ are input values.

More complex generalized structural scheme of IIC described in [5] is shown in Fig. 2.

The input values are $X, \mathrm{Z} 1$ and $\mathrm{Z} 2$, and the output value $Y$. The circuit consists of two parts. The first part contains the blocks that perform basic functions: integrator I, sample-andhold device SH, switches SW1 and SW2 switches. The second part contains auxiliary converters $-\mathrm{CV}_{X}, \mathrm{CV}_{\mathrm{Z} 1}, \mathrm{CV}_{\mathrm{Z} 2}, \mathrm{CI}_{1}$, $\mathrm{CI}_{2}, \mathrm{CO}_{1}, \mathrm{CO}_{2}, . ., \mathrm{CO}_{\mathrm{m}}, \mathrm{BC}$, inverter INV, adder ADD, having transfer coefficients, respectively, $\mathrm{K}_{X}, \mathrm{~K}_{\mathrm{Z1}}, \mathrm{K}_{\mathrm{Z} 2}, \mathrm{~K}_{\mathrm{CI} 1}, \mathrm{~K}_{\mathrm{Cl} 2}$, $\mathrm{K}_{\mathrm{CO} 1}, \mathrm{~K}_{\mathrm{CO} 2}, . ., \mathrm{K}_{\mathrm{COm}}, \beta, \mathrm{K}_{\mathrm{INV}}, \mathrm{K}_{\mathrm{ADD}}$.

The work of the converter is described in [5].

Consider the case where the combination of input and output quantities is the following: $X=X(t)$ is variable value; $Z 1, Z 2$ and $Y$ are constant values.

Similarly to the way it was done in [2] - [5], we can write down in this case the expressions for determining the output quantity $Y$ after the end of the 1st, 2nd and nth conversion cycles:

$$
Y_{1}=K_{X} \prod_{i=1}^{l} K_{i} J_{11}+Y_{0} Q, \quad Y_{2}=K_{X} \prod_{i=1}^{l} K_{i}\left(J_{11} Q+J_{12}\right)+Y_{0} Q^{2}
$$




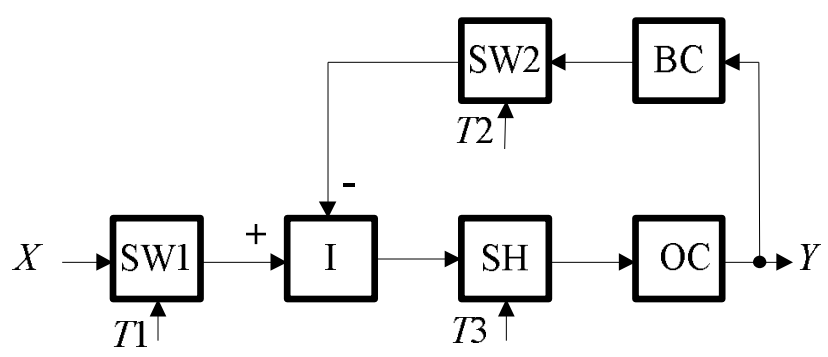

Fig. 1. Simplified generalized structural scheme of iteratively integrating converter.

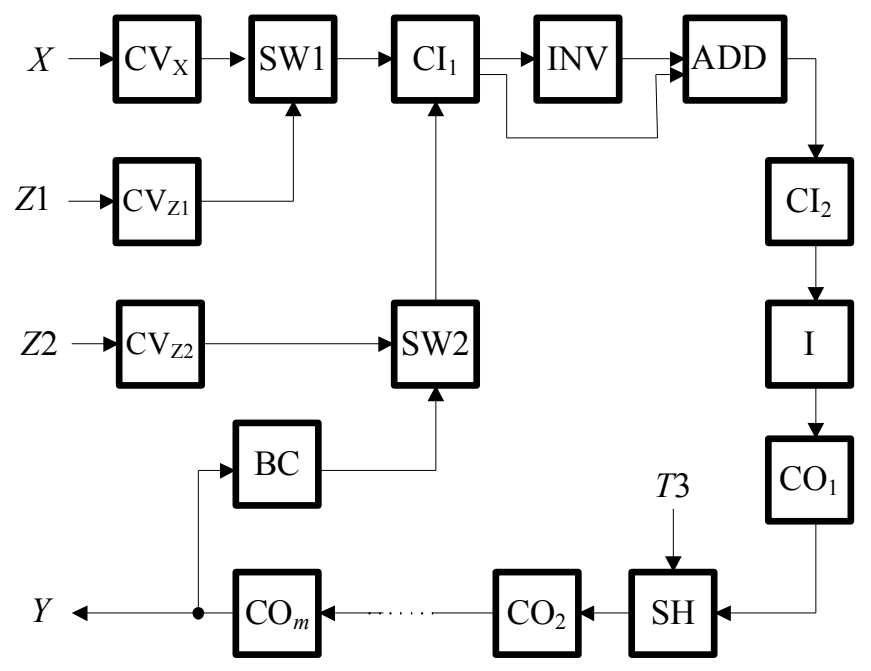

Fig. 2. A generalized structural scheme of iteratively integrating converter.

$$
Y_{n}=K_{X} \prod_{i=1}^{l} K_{i} \sum_{j=1}^{n} J_{1 j} Q^{j-1}+Y_{0} Q^{n}
$$

where $J_{1 j}=\int_{t_{j}}^{t_{j}+Z 1 K_{Z 1}} X(t) d t, t_{j}$ is the current time corresponding to the beginning of the closure of the switch SW1 in the $j$ th conversion cycle.

Here it is assumed that the variable $X=X(t)$ at the input of the converter $\mathrm{CV}_{X}$ and the output value of the block $\mathrm{CI}_{1}$ represent the same function, that is, the converters $\mathrm{CV}_{X}$ and $\mathrm{CI}_{1}$ do not change the shape of the curve $X(t)\left(\mathrm{CV}_{X}\right.$ and $\mathrm{CI}_{1}$ are scale converters).

Suppose that the average value of $X(t)$, for the time that the SW1 switch is closed in each cycle, is unchanged. In this case, the equality $J_{11}=J_{12}=\ldots=J_{1 n}=$ const is observed, and $Z 1 \mathrm{~K}_{\mathrm{Z} 1}$ is equal to or multiples of the period of variation of $X(t)$. Then expression (1) is transformed to the form:

$$
\begin{aligned}
Y_{n}=K_{X} Z 1 K_{Z 1} X_{A V} \prod_{i=1}^{l} K_{i} \sum_{j=1}^{n} J_{1 j} Q^{j-1}+Y_{0} Q^{n} \\
=\frac{K_{X} Z 1 K_{Z !} X_{A V}}{Z 2 K_{Z 2} \beta}\left(1-Q^{n}\right)+Y_{0} Q^{n} .
\end{aligned}
$$

In this case, the output value of the converter in the steady state is determined from the expression:

$$
Y_{\infty}=\frac{K_{X} Z 1 K_{Z !}}{Z 2 K_{Z 2} \beta} X_{A V}
$$

where

$$
X_{A V}=\frac{1}{T} \int_{0}^{T} X(t) d t
$$

is average value of $X(t)$, and $T$ is period of change of $X(t)$.

If the block $\mathrm{CV}_{X}$ or $\mathrm{CI}_{1}$ changes the shape of the input variable curve $X(t)$ (changes the form of the information representation), in particular, at least one of these blocks is a measuring converter of the mean, rms or amplitude values of the input variable $X(t)$, then in this case the expression (2) and (3) are transformed to the form:

$$
\begin{aligned}
& Y_{n}=\frac{\left[X(t) K_{X} K_{1}\right]_{A V} Z 1 K_{Z !}}{Z 2 K_{1} K_{Z 2} \beta}\left(1-Q^{n}\right)+Y_{0} Q^{n}, \\
& Y_{\infty}=\frac{\left[X(t) K_{X} K_{1}\right]_{A V} Z 1 K_{Z !}}{Z 2 K_{1} K_{Z 2} \beta},
\end{aligned}
$$


where $\left[X(t) K_{X} K_{1}\right]_{A V}=\frac{1}{Z 1 K_{Z 1}} \int_{t_{j}}^{t_{j}+Z 1 K_{Z 1}}\left[X(t) K_{X} K_{1}\right] d t$.

If the value $X(t) K_{X} K_{1}=X_{1}+X_{2}$, where $X 1$ is a constant component, and $X_{2}$ is a variable component (for example, pulsations), then the value $\left[X(t) K_{X} K_{1}\right]_{A V}=X_{1}+\left(X_{2}\right)_{A V}$, where $\left(X_{2}\right)_{A V}$ is the average value of the value of $X_{2}$ over time $Z 1 K_{Z 1}$. It's obvious that value $\left(X_{2}\right)_{A V}$ equal to zero if a time $Z 1 K_{Z 1}$ is equal or multiple to the period of the change of the input variable $X(t)$.

As follows from (3) and (4), in the transducer under consideration, the constant component of the input variable $X(t)$ or $\left[X(t) K_{X} K_{1}\right]_{A V}$ is selected, and the time $Z_{1} K_{Z 1}$ of closing the switch SW1 must be equal to or multiples of the period of the input value $X(t)$. Hence two important conclusions about the possibility of using the converter under consideration:

1) The converter under consideration can be used to selection of the useful constant component of the input value $X(\mathrm{t})$ against a background of periodic interference. For this, we have to choose the time of closing the switch SW1 equal to or multiples of the period of the interference.

2) The converter under consideration can be used as a converter for the average values of the input value $X(t)$, for which the time of closing the switch SW1 must be equal to or multiples of the period of the input value $X(t)$. In this case, the converter under consideration as the mean-value converter, what follows from the expressions discussed above in this paper, as well as in [5], has a very high speed. So, for example, if the condition $Q=0$ of the finiteness of the duration of the transient process [5] is satisfied with an error of $10 \%$, then the value of the dynamic error of the output value $\gamma_{n}$ setting will not exceed $0.1 \%$ after three conversion cycles. To obtain the output value of the converter under consideration proportional to the mean rectified value of the input value $X(t)$, it is necessary to carry out a full-wave rectification of the input value $X(t)$. The role of such a rectifier can be performed by the block $\mathrm{CV}_{X}$ or $\mathrm{CI}_{1}$.

Another very interesting conclusion is the following. Let the input value $X(t)$, previously converted by a converter of mean, rms or amplitude values, enter the input of the converter under consideration. The role of such a preliminary converter can be performed by the block $\mathrm{CV}_{X}$ or $\mathrm{CI}_{1}$. In this case, the output value of the converter under consideration is proportional, respectively, to the mean, rms or amplitude value of the input variable $X(t)$. In this case, if the closure time of the switch SW1 is chosen equal to or multiples of the period of the first harmonic of the pulsations, then these pulsations will be largely suppressed (similarly to the suppressing of the periodic disturbance considered above). This makes it possible to use a converter of mean, rms or amplitude values with large pulsations or with a low-pass filter having a small time constant, therefore, with high-speed of convertion. It is interesting to note that in this case a full-wave rectifier (that is, a converter of average values without a low-pass filter) can serve as a converter of average values.

\section{CONCLUSION}

The converter under consideration can be used to selection of the useful constant component of the input value $X(t)$ against a background of periodic interference.

It can be used as a converter for the average values of the input value $X(t)$, for which the time of closing the switch SW1 must be equal to or multiples of the period of the input value $X(t)$. In this case it has a very high speed.

It possible to use the converter under consideration as a converter of mean, rms or amplitude values with large pulsations or with a low-pass filter having a small time constant, therefore, with high-speed of transformation. It is interesting to note that in this case a full-wave rectifier (that is, a converter of average values without a low-pass filter) can serve as a converter of average values.

\section{REFERENCES}

[1] Yu. Tuz, The Structural Methodsof Impruving Accuracy of Measuring Devices, manual, Kyiv. Vyshcha Shkola, 1976. (in Russian).

[2] I. Sergeyev, Research and Development of Integrating Measurement Converters with the Iterative Additive Correction of Errors, Ph. D. (Engineering) Thesis, Kyiv Politechnical Institute, Kyiv, Ukraine, 1978. (in Russian).

[3] Yu. Tuz and I. Sergeyev, "An iterative converter of time interval to the voltage.” Measurement Equipment, no. 7, pp. 15-17, 1976 (in Russian).

[4] I. Sergeyev, "Analysis of the ADC with a dynamic integrator." Measurement Equipment, no. 6, pp. 38-40, 1976 (in Russian).

[5] I. Sergeyev, AVIA-2017: XIII Int. Conf., proc., April 19-21, 2017, Kyiv, 2017, pp. 3.18-3.23. 